\title{
WEAK AND STRONG TYPE ESTIMATES FOR THE COMMUTATORS OF HAUSDORFF OPERATORS
}

\author{
Amjad Hussain And Mudassar AHMED
}

Abstract. This paper addresses weak and strong type Lebesgue space estimates for the commutators of $n$-dimensional Hausdorff operators when the symbol functions belong to Lipschitz space. Strong type estimates for such commutators on classical Morrey spaces are established as well.

Mathematics subject classification (2010): 26D15, 42B35, 42B99, 46E30.

Keywords and phrases: Hausdorff operators, commutators, weak and strong type space, Lipschitz functions, Marcinkiewicz interpolation.

\section{REFERENCES}

[1] V. Burenkov, H. Guliyev And V. Guliyev, Necessary and sufficient conditions for the bounndedness of fractional maximal operators in local Morrey type spaces, J. Comput. Appl. Math. 208 (2007), 280-301.

[2] V. Burenkov, P. JAin And T. TARARYKova, On boundedness of the Hardy operator in Morrey-type spaces, Eurasian Math. J. 2 (2011), 52-80.

[3] J. Chen, D. FAn And J. Li, Hausdorff operators on function spaces, Chin. Ann. Math. 33B (2012), $537-556$.

[4] J. ChEn, D. FAn And S. WANG, Hausdorff operators on Eucleadian spaces, Appl. Math. 28 (2013), $548-564$

[5] J. CHEN AND X. ZHU, Boundedness of multidimensional Hausdorff operators on $H^{1}\left(\mathbb{R}^{n}\right)$, J. Math. Anal. Appl. 409 (2014), 428-434.

[6] M. Christ And L. GRafakos, Best constants for two nonconvolution inequalities, Proc. Amer. Math. Soc. 123 (1995), 1687-1693.

[7] D. FAN AND X. Lin, Hausdorff operator on real Hardy spaces, Analysis 34 (2014), 319-337.

[8] D. FAn AND F. ZhaO, Multilinearf fractional Hausdorff operators, Acta Math. Sinica, 30 (2014), $1407-1421$.

[9] Z. Fu, S. LU AND F. ZhaO, Commutators of $n$-dimensional rough Hardy operators, Sci. China Math. 54 (2011), 95-104.

[10] G. GAO AND H. JiA, Boundedness of commutators of high dimensional Hausdorff operator, J. Function Spaces Appl. 2012 (2012):54120.

[11] G. GAO, X. Wu And W. GuO, Some results for Hausdorff operators, Math. Ineq. Appl. 18 (2015), $155-168$.

[12] G. Gao, X. Wu, A. Hussain, W. Guo, Some estimates of Hausdorff operators, J. Math. Inequal. 9 (2015), 641-651.

[13] G. GAo And Y. Zhong, Some inequalities for Hausdorff operators, Math. Ineq. Appl. 17 (2014), 1061-1078.

[14] L. Grafakos, Classical Fourier Analysis, Second edition, Graduate Texts in Math., Springer, New York, 2008.

[15] A. Hussain And G. Gao, Some new estimates for the commutators of n-dimensional Hausdorff operator, Appl. Math. 29 (2014), 139-150.

[16] A. Hussain AND G. GAO, Multidimensional Hausdorff operators and commutators on Herz-type spaces, J. Ineq. Appl. 2013 (2013):594. 
[17] E. LifLyAND, Boundedness of multidimensional Hausdorff operators on $H^{1}\left(\mathbb{R}^{n}\right)$, Acta Sci. Math. (Szeged) 74 (2008), 845-851.

[18] E. Liflyand, Hausdorff operators on Hardy spaces, Eurasian Math. J. 4 (2013), 101-141.

[19] E. LifLYAND AND A. MIYACHI, Boundedness of the Hausdorff operators in $H^{p}$ spaces, $0<p<1$, Stud. Math. 194 (2009), 279-292.

[20] A. Lerner AND E. LiflyAnd, Multidimensional Hausdorff operators on the real Hardy space, J. Austr. Math. Soc., 83 (2007), 79-86.

[21] E. LiflyAND AND F. MóRICZ, The Hausdorff operator is bounded on the real Hardy space $H^{1}(\mathbb{R})$, Proc. Amer. Math. Soc. 128 (2000), 1391-1396.

[22] X. Lin And L. Sun, Some estimates on the Hausdorff operator, Acta Sci. Math. (Szeged) 78 (2012), $669-681$.

[23] S. Long And J. WANG, Commutators of Hardy operators, J. Math. Anal. Appl. 274 (2002), 626-644.

[24] C. Morrey, On the solutions of quasi-linear elliptic partial differential equations, Trans. Amer. Math. Soc. 43 (1938), 126-166.

[25] M. Paluszynski, Characterization of Besov spaces via the commutator operator of Coifman, Rochberg and Weiss, Ind. Univ. Math. J. 44 (1995), 1-18. 